\title{
Isotope Effects in Proton-transfer Reactions. V.* Deuterium Solvent Isotope Effect on the Racemization of (+)-2-Methyl-3-
} phenylpropionitrile in Methanol

\author{
NILS-ÅKE BERGMAN and LARS MELANDER
}

Department of Organic Chemistry, University of Göteborg and Chalmers University of Technology, Fack, S-402 20 Göteborg 5, Sweden

The deuterium solvent isotope effect on the methoxide-ion catalyzed racemization of $(+)$ 2-methyl-3-phenylpropionitrile and the isotopic $-2-d$ compound has been measured for different mixtures of methanol and methanol-O.d at $60^{\circ} \mathrm{C}$, and the effect has been found to be the same (within the experimental accuracy) for the two substrates. The limiting value of the ratio of the rate constants is $k_{\mathrm{MeOD}} / k_{\mathrm{MeOH}}=$ $2.11 \pm 0.05$. The magnitude of the effect is discussed with reference to the picture obtained earlier for the transition state of this reaction. The usefulness of the magnitude of the solvent isotope effect and the curvature of the isotopeeffect curve as mechanistic tools is discussed briefly.

The primary hydrogen isotope effect for the methoxide-ion catalyzed racemization of 2-methyl-3-phenylpropionitrile has previously been measured and was found to be very weak. ${ }^{1}$ This weak isotope effect was interpreted in terms of a highly unsymmetric transition state, ${ }^{2}$ the proton being almost completely transferred to the methoxide ion. The relatively simple model used for this reaction took no explicit account of the solvation of the methoxide ion and the transition state by methanol molecules. Thus the transition state was considered as a carbanion, formed from the nitrile by abstraction of a proton, and a methanol molecule, formed from the methoxide ion and the abstracted proton.

An attempt has now been made to gain further information about the structure of the transition state by means of the deuterium solvent isotope effect.

* For Part IV of this series, see Ref. 2.

\section{EXPERIMENTAL}

$(+)-2-M e t h y l-3-p h e n y l p r o p i o n i t r i l e$ and -2-d. The preparation of the substrates has been described elsewhere. ${ }^{1}$

Methanol. The drying of the methanol was performed as in Ref. 3, but Linde type 3 A molecular sieves was used in the present case.

Methanol-O-d. Commercial methanol-O-d (CIBA, isotopic purity 99 atom \% D according to NMR) was used without further purification.

Solutions for kinetic runs were prepared as before.1

Kinetic runs were performed on a PerkinElmer 141 polarimeter (in some later runs a Zeiss Old 5 digital polarimeter) in thermostated polarimeter cells of $10.0 \mathrm{~cm}$ length and a volume of $1 \mathrm{ml}$. One run (the first one in $64.2 \%$ MeOD), however, was performed in sealed glass ampoules which were not broken until the polarimetric measurement was made, but, as that method showed no advantages over keeping the sample in the polarimeter cell, the latter method was used in all other runs. All runs were performed at $60.0 \pm 0.1^{\circ} \mathrm{C}$. The evaluation of the rate constants was performed by the method of least squares.

\section{RESULTS}

The results from the measurements of the kinetic solvent isotope effect on the racemization of 2-methyl-3-phenylpropionitrile in mixtures of methanol and methanol. $O-d$ are shown in Table 1. Light as well as heavy substrate was used in the measurements.

The ratios $k_{n} / k_{0}$ between the rate in a solvent with the fractional content $n$ of methanol-O.d and that in ordinary methanol, respectively, refer to experiments with the same isotopic 
Table 1. Summary of the rate-constant data in the racemization of (+)-2-methyl-3-phenylpropionitrile and $-2 \cdot d$ in mixtures of methanol and methanol-O- $d$ with sodium methoxide as base. Substrate concentration $0.15-0.35$ M. Temperature $60.0 \pm 0.1^{\circ} \mathrm{C}$.

\begin{tabular}{|c|c|c|c|c|c|}
\hline Substrate $^{a}$ & $\begin{array}{l}\text { Mol fraction } \\
(n) \text { of } \\
\text { methanol-O-d }\end{array}$ & $\begin{array}{l}\text { Base } \\
\mathbf{M}\end{array}$ & $\begin{array}{l}k \times 10^{6 b} \\
M^{-1} \mathrm{~s}^{-1}\end{array}$ & $k_{n} / k_{0}^{c}$ & $\left(k_{n} / k_{0}\right)_{\mathrm{av}}{ }^{c}$ \\
\hline$+\mathrm{H}$ & 0 & 0.273 & $7.55 \pm 0.27^{d}$ & 1 & 1 \\
\hline$+D$ & 0 & 0.273 & $6.60 \pm 0.19^{d}$ & I & 1 \\
\hline$+\mathbf{H}$ & 0.292 & 0.328 & $9.23 \pm 0.30$ & $1.22)$ & \\
\hline$+D$ & 0.292 & 0.328 & $8.09 \pm 0.27$ & 1.23 & $1.22 \pm 0.01$ \\
\hline$+D$ & 0.292 & 0.320 & $8.04 \pm 0.23$ & $1.22)$ & \\
\hline$+\mathbf{H}$ & 0.483 & 0.311 & $10.88 \pm 0.22$ & 1.44 ) & \\
\hline$+\mathbf{H}$ & 0.483 & 0.310 & $11.04 \pm 0.17$ & 1.46 & \\
\hline$+D$ & 0.483 & 0.313 & $9.37 \pm 0.12$ & 1.42 & $1.43 \pm 0.04$ \\
\hline$+D$ & 0.483 & 0.315 & $9.17 \pm 0.12$ & 1.39 & \\
\hline$+\mathrm{H}$ & 0.642 & 0.306 & $12.06 \pm 0.32$ & 1.60 & \\
\hline$+\mathrm{H}$ & 0.642 & 0.307 & $12.13 \pm 0.32$ & 1.61 & $1.60 \pm 0.01$ \\
\hline$+D$ & 0.642 & 0.307 & $10.50 \pm 0.33$ & $1.59)$ & \\
\hline$+\mathrm{H}$ & 0.990 & 0.310 & $15.40 \pm 0.56$ & $2.04\}$ & \\
\hline$+\mathbf{H}$ & 0.990 & 0.307 & $15.69 \pm 0.39$ & 2.08 & \\
\hline$+\mathbf{H}$ & 0.990 & 0.230 & $15.91 \pm 0.26$ & 2.11 & $2.09 \pm 0.05$ \\
\hline$+\mathrm{H}$ & 0.990 & 0.223 & $16.07 \pm 0.32$ & 2.13 & \\
\hline
\end{tabular}

$a+\mathrm{H}=(+)$-2-Methyl-3-phenylpropionitrile; $+\mathrm{D}=(+)$-2-Methyl-3-phenylpropionitrile-2-d. ${ }^{b} k$ denotes second-order rate constant. Errors include uncertainty in the base concentration. ${ }^{c} k_{n}$ denotes the rate constant for the racemization in a solvent with the fractional content $n$ of deuterium. $k_{0}$ denotes the rate constant for racemization in methanol. $d$ Value from Ref. 1.

composition of the substrate in both cases, i.e., either to ordinary 2-methyl-3-phenylpropionitrile or to its $-2-d$ derivative. There seemed to be no significant difference in $k_{n} / k_{0}$ between the two sets of experiments, and hence the average of $k_{n} / k_{0}$ was taken regardless of the mass of the hydrogen atom of the substrate. In order to obtain the limiting kinetic solvent isotope effect a polynomial was fitted to the experimental points by the method of least squares. The polynomial has the form: $k_{n} / k_{0}=1.000+0.655 n+0.451 n^{2}$, and a graphical representation can be seen in Fig. 1. The value for $n=1$ is $k_{1} / k_{0}=2.1_{08}$, or, in view of the accuracy of the determination in $99 \%$ methanol-O-d, $k_{\mathrm{MeOD}} / k_{\mathrm{MeOH}}=2.11 \pm 0.05$.

From the rate-constant data in Table 1 primary isotope-effect data in different mixtures of methanol and methanol-O-d can be obtained. These results are shown in Table 2.

\section{DISCUSSION}

The plot of equilibrium or rate constants versus the isotopic composition of the medium is generally curved. This was detected long ago

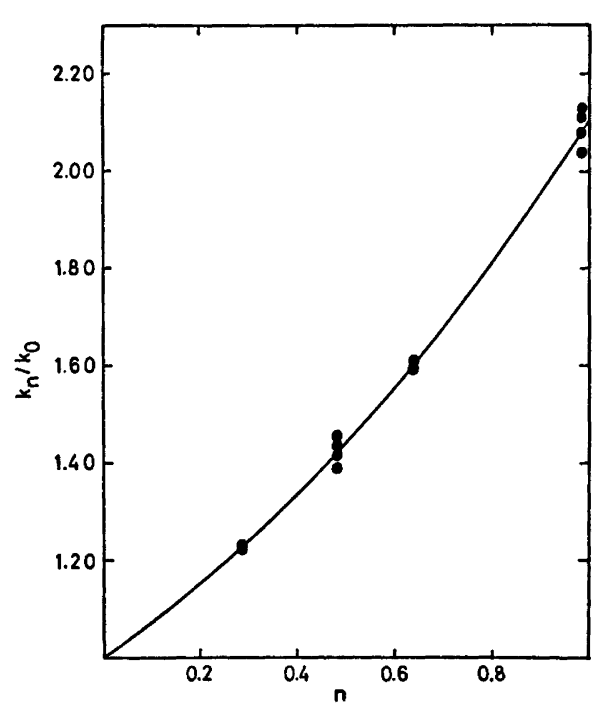

Fig. 1. Plot of $k_{n} / k_{0}$ against isotope composition of the medium. The filled circles denote experimental values. The curve represents the polynomial $k_{n} / k_{0}=1.000+0.655 n+0.451 n^{2}$. Temperature $60.0 \pm 0.1{ }^{\circ} \mathrm{C}$.

Acta Chem. Scand. A 28 (1974) No. 7 
Table 2. Primary kinetic isotope-effect data for different mixtures of methanol and methanol$O-d$ as solvent. Temperature $60.0 \pm 0.1^{\circ} \mathrm{C}$.

\begin{tabular}{ll}
\hline $\begin{array}{l}\text { Solvent composition, } \\
\text { mol fraction methanol-O-d }\end{array}$ & $k_{\mathrm{D}} / k_{\mathrm{H}}{ }^{a}$ \\
\hline & \\
\hline 0 & $0.87 \pm 0.04^{b}$ \\
0.292 & 0.87 \\
0.483 & 0.85 \\
0.642 & 0.87 \\
\hline
\end{tabular}

a Ratio of rate constant for heavy and light substrate, respectively. ${ }^{b}$ From Ref. 1 .

by La Mer and Chittum in an investigation of the variation of the dissociation constant of acetic acid in mixtures of $\mathrm{H}_{2} \mathrm{O}$ and $\mathrm{D}_{2} \mathrm{O}$. Since then a great number of results concerning solvent isotope effects in mixtures of $\mathrm{H}_{2} \mathrm{O}$ and $\mathrm{D}_{2} \mathrm{O}$ have been published. The observed phenomena have been thoroughly interpreted in terms of the mechanism of the reactions, and the use of the curvature of a solvent isotopeeffect plot and the limiting solvent isotope effect as mechanistic tools has been discussed. ${ }^{5-8}$ The investigations have mainly been concerned with reactions in mixtures of $\mathrm{H}_{2} \mathrm{O}$ and $\mathrm{D}_{2} \mathrm{O}$, but in recent years some investigations of the variation of the solvent isotope effect in mixtures of methanol and methanol-O-d have appeared.' Solvent isotope effects in other alcohols have also been investigated. ${ }^{10,11}$

The discussion of solvent isotope effects and their variation with the isotopic composition of the solvent is most conveniently carried through in terms of fractionation factors for the transition state (in the case of kinetic solvent isotope effects) and for the initial state. ${ }^{5,6}$ The fractionation factor for a particular site in a molecule is defined as the ratio of its deuterium/protium ratio and that in the bulk of the solvent. In the present case the hydrogen in the reactant carbon acid and the hydrogen in transit in the transition state are not avail- able for equilibration, because there is no way for them to exchange with the medium except via the reaction being studied. The only exchangeable hydrogen in addition to that in the bulk of the solvent occurs in the methanol molecules solvating the reactant methoxide ion and in those solvating the transition state. (The reactant nitrile can hardly perturb the surrounding solvent to such an extent that appreciable fractionation takes place.)

The only fractionation factors $(\mathrm{D} / \mathrm{H})_{\text {solutel }}$ $(\mathrm{D} / \mathrm{H})_{\text {solvent }}$ to be taken into account in the following are thus those in which $(\mathrm{D} / \mathrm{H})_{\text {solute }}$ refers to the isotopic composition of methanol solvating either the methoxide ion or the transition state, respectively. The former fractionation factor has been determined to $\phi_{\text {Mieo }}=\mathbf{0 . 7 3 _ { \text { o } }}$ at $33.5^{\circ} \mathrm{C},{ }^{\text {,a }}$ and, assuming a pure zero-pointenergy effect, the value at $60^{\circ} \mathrm{C}$ would be $0.75_{4}$, which will be used throughout the present paper.

Reaction Scheme 1 shows the present model of the proton-abstraction reaction. $\mathrm{RH}$ denotes the (+)-2-methyl-3-phenylpropionitrile, and $m$ and $p$ are the numbers of methanol molecules solvating the methoxide ion and the transition state, respectively.

In terms of fractionation factors, the ratio between the rate constant for the reaction in a solvent with the fractional deuterium content $n$ and the rate constant for the same reaction in non-deuterated solvent is given by ${ }^{\circ}$

$k_{n} / k_{0}=\left(1-n+n \phi^{\neq} \neq\right)^{p} /\left(1-n+n \phi_{\mathrm{MeO}}\right)^{m}$

Assuming a value of $\phi \neq$ between 0.75 and 1.0 , the maximum limiting isotopic rate ratio is given by

$k_{1} / k_{0}=1 / 0.754^{m}$

This ratio equals $1.326,1.759,2.333$ for $m=1$, 2,3 , respectively. Because of the magnitude of the experimental limiting rate ratio $\left(k_{1} / k_{0}=\right.$ $2.11 \pm 0.05$ ) obtained in this investigation, $m=3$ seems most likely. This is also in agreement with results obtained in similar investigations

$$
\begin{gathered}
\mathrm{RH} \cdot \mathrm{CH}_{3} \mathrm{O}^{-}\left(\mathrm{CH}_{3} \mathrm{OH}\right)_{m}=\left[\mathrm{R} \cdots \mathrm{H} \cdot \mathrm{OCH}_{3}\left(\mathrm{CH}_{3} \mathrm{OH}\right)_{p}\right]^{\mathrm{F}^{-}} \cdot(m-p) \mathrm{CH}_{3} \mathrm{OH} \longrightarrow \text { products } \\
\mathrm{R}=\mathrm{O}-\mathrm{CH}_{2}-\mathrm{CH}_{\mathrm{CN}}^{-\mathrm{CH}_{3}^{-}}
\end{gathered}
$$

Scheme 1.

Acta Chem. Scand. A 28 (1974) No. 7 
Table 3. Comparison of experimentally determined $k_{n} / k_{0}$ values for different $n$ 's with values from trial calculations. $\phi_{\mathrm{MeO}}=0.75_{4} \cdot k_{1} / k_{0}=2.1_{08}$.

\begin{tabular}{lllllll}
\hline$n$ & $\left(k_{n} / k_{0}\right)_{11}{ }^{a}$ & $\left(k_{n} / k_{0}\right)_{21}$ & $\left(k_{n} / k_{0}\right)_{22}$ & $\left(k_{n} / k_{0}\right)_{32}$ & $\left(k_{n} / k_{0}\right)_{33}$ & $\left(k_{n} / k_{0}\right)_{\exp }$ \\
\hline 0 & 1 & 1 & 1 & 1 & 1 & 1 \\
0.292 & 1.26 & 1.23 & 1.23 & 1.21 & 1.21 & $1.22 \pm 0.01$ \\
0.483 & 1.46 & 1.41 & 1.41 & 1.39 & 1.39 & $1.43 \pm 0.04$ \\
0.642 & 1.64 & 1.59 & 1.59 & 1.57 & 1.57 & $1.60 \pm 0.01$ \\
0.990 & 2.09 & 2.09 & 2.09 & 2.09 & 2.09 & $2.09 \pm 0.05$ \\
1 & 2.11 & 2.11 & 2.11 & 2.11 & 2.11 & $2.11^{b}$ \\
$\phi \neq c$ & 1.59 & 1.20 & 1.09 & 0.95 & 0.97 & \\
\hline
\end{tabular}

${ }^{a}$ Subscripts denote the assumed values of $m$ and $p$, respectively. ${ }^{b}$ Extrapolated value. ${ }^{c} \phi \neq=$ $\left[2.1_{06}\left(0.75_{4}\right)^{m}\right]^{1 / P}$.

of methoxide-ion catalyzed reactions. ${ }^{\text {cc }}$ The existence of three lone pairs of electrons in the oxygen part of $\mathrm{MeO}^{-}$also makes such a solvation number plausible. Irrespective of the number $p$, this assumption leaves a very small interval for the fractionation factor $\phi \neq$, because $\phi \neq p=2.1_{06}(0.75)^{3}=0.90$.

Some trial calculations were undertaken in order to see whether it is possible to deduce some definite values for $m$ and $p$ from the results obtained in this investigation. The resulting figures are gathered in Table 3 together with the experimental values of $k_{n} / k_{0}$. The stated values for different $m$ and $p$ are calculated from the limiting isotopic rate ratio $k_{1} / k_{0}=2.1_{06}$ and $\phi_{\mathrm{MeO}}=0.75_{4}$.

As can be seen from Table 3, high accuracy in the experimental $k_{n} / k_{0}$ values is required to discriminate between different values of $m$ and $p$. With the results obtained here this is not possible. The calculated values for $m=2, p=1$ or 2 seem to agree very well with the experimentally determined values, but it is doubtful whether any value of $\phi \neq$ above unity is reasonable. Inescapable facts are that the methoxide ion has to be solvated by methanol molecules and that the most probable number of solvating molecules is three.

It has been suggested that proton-transfer reactions of the present type, showing very weak primary isotope effects, should proceed via a pre-equilibrium, in the present case followed by a rate-determining racemization step. Such a mechanism would easily explain the weak dependence upon isotopic mass. According to Cram ${ }^{12}$ a possible reaction sequence would be as in Scheme 2.

The expression given above for $k_{n} / k_{0}$ would still be valid for the two cases $k_{-1} \gg k_{2}$ and $k_{-1} \ll k_{2}$ if $p$ denotes the solvation number of the rate-determining transition state (transition state 2 and transition state 1, respectively). There is no obvious difference in the most likely assumptions concerning the solvation numbers in the two cases. The charge on the carbanion must be more fully developed in transition state 2 than in transition state 1. Irrespective of the assumption of one or more kinds of methanol molecules solvating the transition state, the limiting solvent isotope effect leaves little room for a high power of fractionation factors appreciably inferior to unity. Introduction of more fractionation factors for the transition state, accounting for possible solvation of the charge on the carbanion, makes therefore little sense.

It may be questioned whether there is a real difference between the strongly endothermal single-step model ${ }^{2}$ and the one with two reaction steps, the second of which is ratedetermining. In the way $\operatorname{Cram}^{12}$ writes it, cf. Scheme 2, the rate-determining process is nothing but a separation of the two reaction products. The reaction coordinate has conse-

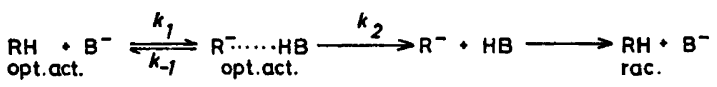

Scheme 2. 
quently the same nature in both models. In such a case there is little hope of distinguishing between the models.

Transition-state fractionation factors are thought to be intermediate between reactant and product fractionation factors and can possibly be used to give some information about the transition-state structure. ${ }^{8}$ Let $x$ be some measure of the degree of resemblance of the "methanolic" part of the transition state to the bulk solvent, thus $0 \leq x \leq 1$. Then it may be assumed that $\phi \neq=\phi_{\mathrm{MeO}^{1-x} \text {. }}$

The limiting kinetic solvent isotope effect $k_{1} / k_{0}$ is thus related to $x$ through the relationship:

$k_{1} / k_{0}=\phi_{\mathrm{MeO}}^{(1-x) p} / \phi_{\mathrm{MeO}}^{m}=\phi_{\mathrm{MeO}}^{p(1-x)-m}$

Assuming $m=3$ and $p=2$ the calculated value of $x$ for $k_{1} / k_{0}=2.1_{08}$ is 0.82 , and $m=p=3$ gives $x=0.88$. These values are somewhat smaller than the Brønsted $\beta$ which has been estimated previously ${ }^{1}$ at about unity. The latter value was thought to reflect the product-like nature of the transition state for this reaction.

The interpretation of kinetic solvent isotope effects in simple terms of transition-state symmetry is perhaps not so straight-forward..$^{13}$ This is also underlined by some collected data from the present literature on the magnitude of primary kinetic isotope effects and solvent isotope effects for methoxide-catalyzed protonabstraction reactions in methanol, given in Table 4. The reactions in Table 4, except for those of 2-nitropropane and phenyl 2,2-diphenylcyclopropyl ketone, are all thought to be endothermal, which can be concluded from the $\mathrm{p} K$ values of the compounds relative to that of methanol. It is hard to see any correlation between the strength of the primary kinetic isotope effect and that of the kinetic solvent isotope effect. The most striking feature of the presented data is perhaps that several examples are found where the primary isotope effect is very strong, indicating a fairly symmetric transition state, but still the kinetic solvent isotope effect has almost reached its maximum value corresponding to $m=3$.

The primary kinetic isotope effect for 2methyl-3-phenylpropionitrile in different mixtures of methanol and methanol-O-d (Table 2) shows no detectable variation. The values of $k_{\mathrm{D}} / k_{\mathrm{H}}$ for the three mixtures in Table 2 all agree within the experimental errors with the value found for undeuterated methanol.1

This constancy of the primary isotope effect in different mixtures of methanol and methanol$O-d$ also implies that the equilibrium constant for the exchange equilibrium between nitrile and methanol should be expected to have a nearly constant value. ${ }^{18}$ This rather reasonable result is at variance with the results in Ref. 3, where a supposed variation of the $K_{\mathrm{H}} / K_{\mathrm{D}}$ ratio with the deuterium content of the medium was found.

Acknowledgements. It is a pleasure to acknowledge the interesting discussions between Dr. John Albery and one of us (L. M.) during a most pleasant visit at Oxford University. Financial support from The Swedish Natural Science Research Council is gratefully acknowledged.

Table 4. Methoxide-catalyzed proton-abstraction reactions in methanol. A comparison between primary kinetic isotope effects and kinetic solvent isotope effects.

\begin{tabular}{|c|c|c|c|c|}
\hline Substrate & $\begin{array}{l}\text { Temp. } \\
{ }^{\circ} \mathrm{C}\end{array}$ & $\begin{array}{l}\text { Isotopic } \\
\text { primary } \\
k_{\mathrm{D}} / k_{\mathrm{H}}\end{array}$ & $\begin{array}{l}\text { rate ratio } \\
\text { solvent } \\
k_{\mathrm{MeOD}} / k_{\mathrm{MeOH}}\end{array}$ & Reference \\
\hline \multirow{4}{*}{$\begin{array}{l}\text { 2-Nitropropane } \\
\text { 2-( }, N \text {-Dimethylcarbox- } \\
\text { amido)-9-methylfluorene } \\
\text { 9-Methylfluorene } \\
\text { Phenyl 2,2-diphenyl- } \\
\text { cyclopropyl ketone } \\
\text { 1-H-perfluoroheptane } \\
\text { 2-Methyl-3-phenyl- } \\
\text { propionitrile } \\
\text { Pentafluorobenzene }\end{array}$} & 24.8 & 0.135 & 2.28 & $9 \mathrm{c}$ \\
\hline & $\begin{array}{l}24.9 \\
44.7\end{array}$ & $\begin{array}{l}0.15 \\
0.19\end{array}$ & $\begin{array}{l}2.3 \\
2.2\end{array}$ & $\begin{array}{l}14 \\
15\end{array}$ \\
\hline & $\begin{array}{l}50 \\
60\end{array}$ & $\begin{array}{l}0.286 \\
0.48\end{array}$ & $\begin{array}{l}2.08 \\
1.52\end{array}$ & \multirow{2}{*}{$\begin{array}{l}10 \\
16 \\
1 \text { and present } \\
\text { investigation } \\
17\end{array}$} \\
\hline & $\begin{array}{l}60 \\
25.02\end{array}$ & $\begin{array}{l}0.87 \\
1.0\end{array}$ & $\begin{array}{l}2.11 \\
2.3\end{array}$ & \\
\hline
\end{tabular}

Acta Chem. Scand. A 28 (1974) No. 7 


\section{REFERENCES}

1. Melander, L. and Bergman, N.-A. Acta Chem. Scand. 25 (1971) 2264.

2. Bergman, N.-Å., Saunders, W. H., Jr. and Melander, L. Acta Chem. Scand. 26 (1972) 1130 .

3. Bergman, N.-A. Acta Chem. Scand. 25 (1971) 1517.

4. La Mer, V. K. and Chittum, J. P. J. Amer. Chem. Soc. 58 (1936) 1642.

5. Gold, V. a. Trans. Faraday Soc. 56 (1960) 255; b. Advan. Phys. Org. Chem. 7 (1969) 259.

6. Kresge, A. J. Pure Appl. Chem. 8 (1964) 243.

7. Albery, W. J. and Davies, M. H. J. Chem. Soc. Faraday Trans. 1 (1972) 167.

8. Showen, R. L. Progr. Phys. Org. Chem. 9 (1972) 275.

9. Gold, V. and Grist, S. J. Chem. Soc. B (1971) a. 1665; b. 2272; c. 2282; d. 2285 .

10. Levin, J.-O. and Rappe, C. Chem. Scr. 1 (1971) 233.

11. Levin, J.-O. Chem. Scr. 4 (1973) 85.

12. Cram, D. J. Fundamentals of Carbanion Chemistry, Academic, New York and London 1965 , pp. $28-29$.

13. Gold, V. and Grist, S. J. Chem. Soc. Perkin Trans. 1 (1972) 89.

14. Ford, W. T., Graham, E. W. and Cram, D. J. J. Amer. Chem. Soc. 89 (1967) 4661.

15. Streitwieser, A., Jr., Hollyhead, W. B., Pudjaatmaka, A. H., Owens, P. H., Kruger, T. L., Rubenstein, P. A., MacQuarrie, R. A., Brokaw, M. L., Chu, W. K. C. and Niemeyer, H. M. J. Amer. Chem. Soc. 93 (1971) 5088.

16. Andreades, S. J. Amer. Chem. Soc. 86 (1964) 2003.

17. Streitwieser, A., Jr., Hudson, J. A. and Mares, F. J. Amer. Chem. Soc. 90 (1968) 648.

18. Melander, L. Acta Chem. Scand. 25 (1971) 3821.

Received April 8, 1974. 